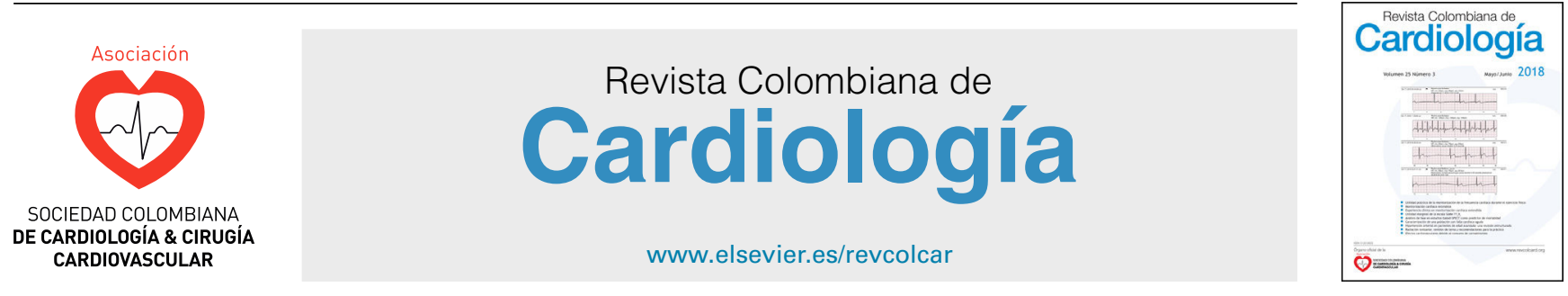

CARDIOLOGÍA DEL ADULTO - ARTÍCULO ORIGINAL

\title{
Concordancia de la motilidad segmentaria evaluada por ecocardiograma transtorácico y resonancia magnética cardíaca en pacientes con infarto agudo de miocardio
}

\author{
Alberto Cadena-Bonfanti ${ }^{a}$, Henry J. González-Torres ${ }^{\mathrm{b}, *}$, \\ Bayron Valdes-Casas ${ }^{\mathrm{b}}$ y Fabián Ruiz-Pla ${ }^{\mathrm{a}}$ \\ a Servicio de Cardiología, Clínica de la Costa, Barranquilla, Colombia \\ b Universidad Simón Bolívar, Facultad de Ciencias de la Salud, Barranquilla, Colombia
}

Recibido el 19 de octubre de 2017; aceptado el 6 de abril de 2018

Disponible en Internet el 4 de octubre de 2018

\author{
PALABRAS CLAVE \\ Ecocardiografía; \\ Resonancia \\ magnética cardiaca; \\ Infarto agudo de \\ miocardio
}

\begin{abstract}
Resumen
Introducción: La evaluación de la contractilidad segmentaria es un marcador importante para determinar la extensión de la enfermedad coronaria manifestada a través de un evento isquémico cardiaco. La resonancia magnética cardíaca es el patrón estándar para evaluar dicha motilidad; sin embargo, debido al elevado número de exámenes, la disponibilidad de equipos de resonancia magnética y el costo de los exámenes de resonancia magnética, el uso del ecocardiograma transtorácico es el método diagnóstico de elección.

Objetivo: Evaluar la concordancia de la evaluación de la motilidad de diferentes segmentos miocárdicos y de agrupación de segmentos por territorios de irrigación arterial obtenidos por resonancia magnética cardiaca y ecocardiograma transtorácico en pacientes hospitalizados por infarto agudo de miocardio.

Métodos: A un grupo de pacientes que llegaron al servicio de emergencia por infarto agudo de miocardio, se les realizó ecocardiograma transtorácico y resonancia magnética cardiaca y se compararon los resultados. La resonancia magnética cardiaca fue el valor de referencia a comparar para fracción de eyección y contractilidad segmentaria. Las pruebas comparativas se hicieron a un $95 \%$ de confianza mediante el coeficiente de Kappa-Cohen para evaluar la concordancia entre las medidas.

Resultados: No se encontraron diferencias entre la fracción de eyección del ventrículo izquierdo medida por ecocardiograma transtorácico y resonancia magnética cardiaca. Los territorios irrigados por las arterias coronarias descendente anterior y circunfleja tenían una concordancia mediana y buena. En aquellos asociados con la arteria coronaria derecha la concordancia fue baja y media.
\end{abstract}

\footnotetext{
* Autor para correspondencia.

Correo electrónico: hgonzalez11@unisimonbolivar.edu.co (H.J. González-Torres)
} 


\section{KEYWORDS}

Transthoracic echocardiogram; Cardiac magnetic resonance; Acute myocardial infarction
Conclusiones: La evaluación de la fracción de eyección por ecocardiograma transtorácico no difiere de la evaluación por resonancia magnética cardiaca. En la evaluación de la motilidad segmentaria, la resonancia magnética cardiaca es mejor que el ecocardiograma.

(c) 2018 Sociedad Colombiana de Cardiología y Cirugía Cardiovascular. Publicado por Elsevier España, S.L.U. Este es un artículo Open Access bajo la licencia CC BY-NC-ND (http:// creativecommons.org/licenses/by-nc-nd/4.0/).

\section{Comparison of segmental contractility evaluated by transthoracic echocardiogram} and cardiac magnetic resonance in patients with acute myocardial infarction

\begin{abstract}
Introduction: The evaluation of segmental contractility is an important marker to determine the extent of the coronary disease manifested by an ischaemic event. Cardiac magnetic resonance is the standard pattern to evaluate this contractility. However, due to lower availability of magnetic resonance equipment, as well as the increased cost of magnetic resonance tests, the use of the transthoracic echocardiogram is the diagnostic method of choice.

Objective: The aim of the study is to compare the evaluation of the motility of different myocardial segments and segments grouped by coronary artery supply territories obtained by cardiac magnetic resonance and transthoracic electrocardiogram performed on patients admitted to hospital with acute myocardial infarction.

Methods: A cardiac magnetic resonance and transthoracic electrocardiogram was performed on a group of patients that arrived in the Emergency Department due to an acute myocardial infarction, and the results were compared. The cardiac magnetic resonance was the reference value to compare for the ejection fraction and segmental contractility. Comparative tests were performed with $95 \%$ confidence limits using a Kappa-Cohen coefficient to evaluate the agreement between the measurements.

Results: No differences were found between the left ventricular ejection fraction measured by cardiac magnetic resonance and transthoracic electrocardiogram. There was average and good agreement in the coronary and circumflex artery supply territories, respectively. In those associated with the right coronary artery, the concordance was low and average.

Conclusions: There was no difference between the evaluation of the ejection fraction by transthoracic electrocardiogram or that by cardiac magnetic resonance. In the evaluation of segmental motility, cardiac magnetic resonance was better than transthoracic electrocardiogram.

(C) 2018 Sociedad Colombiana de Cardiología y Cirugía Cardiovascular. Published by Elsevier España, S.L.U. This is an open access article under the CC BY-NC-ND license (http:// creativecommons.org/licenses/by-nc-nd/4.0/).
\end{abstract}

\section{Introducción}

Los efectos de la isquemia coronaria sobre el miocardio ventricular son ampliamente conocidos desde hace casi un siglo $^{1-3}$. A partir de entonces, la valoración de la contractilidad segmentaria se toma como marcador de la extensión de la enfermedad coronaria ${ }^{4}$. En la práctica moderna el modelo de reporte más utilizado es el de 17 segmentos, el cual guarda correlación con la irrigación coronaria ${ }^{5}$.

La irrigación sanguínea del ventrículo izquierdo (VI) se realiza por tres arterias coronarias. La arteria descendente anterior izquierda (LDA, por su sigla en inglés) irriga los segmentos 1, 2, 7, 8, 13, 14 y 17; la arteria coronaria derecha (RCA, por su sigla en inglés) los segmentos 3, 4, 9, 10, y 15y la arteria circunfleja izquierda (LCX, por su sigla en inglés) los segmentos $5,6,11,12$, y 16 . A la agrupación de los segmentos se le conoce como territorios ${ }^{6}$ (fig. 1).

Existen varios métodos para valoración de la función miocárdica; la información derivada de estos estudios genera información pronóstica e influencia el tratamiento. El patrón

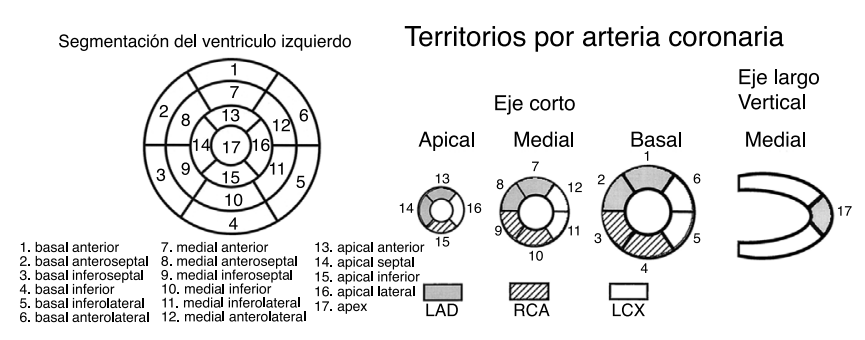

Figura 1 Segmentación del ventrículo izquierdo y territorios por arteria coronaria.

Fuente: Cerqueira et al., Circulation. 2002;105(4):539-42 ${ }^{5}$.

estándar para la valoración de la función y de los volúmenes del miocardio ventricular es la resonancia magnética cardiaca ${ }^{7}$. En la actualidad el método predilecto y con más difusión en la valoración de la contractilidad miocárdica es el ecocardiograma transtorácico. Sin embargo, tiene limitaciones propias de la técnica, como mala ventana que impide 
la visualización del miocardio ventricular, alteraciones geométricas relacionadas que alteran el cálculo de volúmenes y la obtención de imágenes por fuera del eje anatómico, la cual dificulta la estimación de volúmenes y la valoración de los segmentos en los que se divide el miocardio ventricular ${ }^{8}$. Además, la valoración de infarto por ecocardiograma transtorácico solamente basado en parámetros de motilidad puede subestimar o sobrestimar la cantidad de miocardio infartado ${ }^{5,9}$.

La resonancia magnética cardíaca posee ventajas, como la posibilidad de obtención de imágenes en cualquier plano, sobre la ecocardiografía; pese a ello no cuenta con la versatilidad de la ecocardiografía, requiere más preparación técnica, mayor tiempo para su ejecución y más colaboración del paciente, lo cual dificulta su uso en situaciones de inestabilidad hemodinámica. La valoración de fracción de eyección y de motilidad segmentaria por ambas técnicas en el contexto de infarto agudo de miocardio, no tiene una correlación significativa ${ }^{7,10,11}$. A pesar de las ventajas de la valoración de la motilidad por resonancia, no es una técnica muy difundida en la valoración inicial de infarto de miocardio.

Dentro de las posibles desventajas de la resonancia magnética cardiaca están los marcapasos, que anteriormente suponían un inconveniente, que ha sido superado por los nuevos marcapasos. Otro posible inconveniente es la colaboración del paciente y la claustrofobia, no obstante, hay métodos para reducir dicha fobia, lo cual no se considera un impedimento común y no ocurrió en este estudio.

De otro lado, el ecocardiograma es el método diagnóstico más utilizado para la evaluación del infarto agudo de miocardio; sin embargo se presentan limitaciones inherentes a la técnica ${ }^{12}$, de ahí que incluya en el estudio del infarto de miocardio una herramienta como la resonancia magnética cardiaca, dado que no posee limitaciones en la adquisición de planos y evita las dificultades técnicas de la ecocardiografía previamente mencionadas ${ }^{11}$.

Dentro de las variables de importancia en la evaluación del infarto de miocardio, se encuentra la fracción de eyección, la cual es un importante marcador pronóstico en infarto de miocardio, pero no existe un consenso acerca de la técnica a utilizar para su valoración ${ }^{5,13}$, aunque se favorece el uso de la ecocardiografía por la difusión y disponibilidad de la técnica. En el contexto de síndrome coronario agudo la contractilidad segmentaria se usa como marcador de daño miocárdico, de modo que, en nuestro centro se compara la concordancia de motilidad segmentaria entre ambos métodos en pacientes con síndrome coronario agudo.

Dado que el método de elección en la práctica diaria es el ecocardiograma transtorácico, este estudio determinó la concordancia de la motilidad por segmentos del miocardio ventricular evaluado por ecocardiograma transtorácico y por resonancia magnética cardiaca en pacientes que han sido hospitalizados por infarto agudo de miocardio, teniendo en cuenta la irrigación de territorio por arteria coronaria, determinando si existe diferencia entre las mediciones hechas por ambos métodos.

\section{Materiales y métodos}

Inicialmente, se implementó un reporte electrónico para ecocardiograma y resonancia magnética cardíaca con registro de la motilidad segmentaria en el modelo de 17 segmentos ${ }^{5,14}$. Posterior a ello se hizo un mes de entrenamiento para la familiarización en el uso del reporte.

Entre enero a julio de 2015, posterior al periodo de entrenamiento y familiarización del uso del reporte electrónico, se buscaron en la base de datos local los pacientes ingresados con diagnóstico de síndrome coronario agudo, a quienes se les hubiera practicado ecocardiograma y resonancia magnética cardíaca, en un lapso de tiempo no mayor de siete días entre ambos estudios.

Los estudios de ecocardiografía (Vivid 7) y resonancia magnética cardíaca (Siemens Avanto $1.5 \mathrm{~T}$ ) fueron hechos por operadores diferentes para evitar sesgos en el reporte de resultados. Los ecocardiografistas cumplían con el tiempo de entrenamiento recomendado por la ASECHO (American Society of Echocardiography) y más de 5 años de práctica al momento del estudio ${ }^{15,16}$. Posteriormente se comparó la motilidad segmentaria entre ambos métodos cotejando, segmento por segmento, la correlación de motilidad, considerándolo tal como lo describe la guía tanto para resonancia magnética cardiaca como para ecocardiograma transtorácico ${ }^{4}$ (tabla 1 ).

A cada uno de los segmentos se les tomaron las medidas de tendencia central comparando cada uno de los ellos, los cuales se agruparon por arteria asociada. A estos bloques se les aplicó estadística descriptiva y gráficos básicos ${ }^{17}$. Se evaluó inicialmente la edad por género y el porcentaje de fracción de eyección ventricular izquierda (FEVI) por género, para descartar algún posible sesgo causado por estas variables.

Para evaluar la edad y FEVI por género se realizó una comparación de medias (alfa: 95\%). Posteriormente se hizo un análisis de concordancia mediante el coeficiente kappa de Cohen así como sus intervalos de confianza (IC) ${ }^{14,15}$. Así mismo, se compararon los segmentos asociados por irrigación arterial tanto en la resonancia magnética cardiaca como en el ecocardiograma transtorácico ${ }^{12}$.

\section{Resultados}

Se evaluaron 49 pacientes, de los cuales $53 \%$ pertenecían al sexo femenino y $47 \%$ al masculino. La edad promedio del grupo fue de $67 \pm 10$ años; para el sexo femenino fue de $67 \pm 10$ años, mientras para el masculino fue de $68 \pm 10$ años. No se encontraron diferencias significativas entre las edades por sexo (t: $-0,45 ; \mathrm{p}>0,05)$ (tabla 2).

En cuanto a la FEVI, el promedio para el ecocardiograma transtorácico global fue de $41,6 \pm 13 \%$ y para la resonancia magnética cardiaca global de $39,5 \pm 13,2 \%$, diferencia no significativa $(p>0,05)$. En cuanto al sexo masculino

Tabla 1 Interpretación del coeficiente kappa de Cohen

\begin{tabular}{lll}
\hline $\begin{array}{l}\text { Valor de } \\
\text { kappa }\end{array}$ & Nivel de acuerdo & $\begin{array}{l}\text { \% de datos } \\
\text { que son } \\
\text { confiables }\end{array}$ \\
\hline $0-0,20$ & Ninguno (none) & $0-4 \%$ \\
$0,21-0,39$ & Mínimo (minimal) & $4-15 \%$ \\
$0.40-0,59$ & Débil (weak) & $15-35 \%$ \\
$0,60-0,79$ & Moderado (moderate) & $35-63 \%$ \\
$0,80-0,90$ & Fuerte (strong) & $64-81 \%$ \\
$>0,90$ & Casi perfecto (almost perfect) & $82-100 \%$ \\
\hline
\end{tabular}

Fuente: McHugh M. Biochem Medica. 2012;34(2):276-82 ${ }^{18}$. 
Tabla 2 Comparación de la fracción de eyección por género e imagenología utilizada

\begin{tabular}{llll}
\hline Género & FEVI EcoTT $(\mathrm{n})$ & FEVI-resonancia magnética cardiaca $(\mathrm{n})$ & $\mathrm{p}$-valor \\
\hline Masculino & $45,4 \pm 15,7 \%(23)$ & $40,3 \pm 15,7 \%(23)$ & 0,27 \\
Femenino & $57,5 \pm 7,2 \%(26)$ & $56,8 \pm 12,6 \%(26)$ & 0,79 \\
\hline
\end{tabular}

FEVI: fracción de eyección del ventrículo izquierdo; ECO-TT: ecocardiograma transtorácico; n): Número de sujetos; $p$-valor: valor de significancia estadística.

Fuente: Datos del estudio.

la FEVI medida por ecocardiograma transtorácico fue más alta que la obtenida por resonancia magnética cardiaca, sin embargo, la diferencia no fue significativa ( $p>0,05)$; para el sexo femenino no se encontró diferencia $(p>0,05)$.

\section{Comparativo entre arterias}

\section{Arteria descendente anterior izquierda}

El territorio irrigado por la arteria descendente anterior izquierda (su sigla en inglés LAD), presentó una casi perfecta concordancia en lo referente a territorio (k: 77,14 IC $23 \%)$. No obstante, para los segmentos mediales, el valor de kappa fue más bajo que el promedio del territorio, siendo k: $68,9 \%$, pero aun estando dentro del rango considerado sustancial (k: 0,6-0,8) (fig. 2).

En los segmentos irrigados por la LAD, el que presentó mayor variación de la concordancia fue el segmento anterior medio, que tuvo una subestimación importante en la clasificación de la motilidad segmentaria; tres pacientes que tenían motilidad normal por ecocardiograma transtorácico fueron tipificados con hipoquinesia para este segmento por resonancia magnética cardiaca. Otro segmento con un valor elevado en subestimación de la motilidad fue el anterior apical con un $10 \%$.

\section{Arteria circunfleja izquierda}

Para el territorio de la arteria circunfleja izquierda (LCX por su sigla en inglés), se encontró que la coincidencia de los resultados obtenidos por ECO-TT con los de resonancia magnética cardiaca fue del $86 \pm 47 \%$; sin embargo, al evaluar la concordancia con el coeficiente kappa, el resultado fue de $63 \%$ IC $10,8 \%$, siendo moderado (fig. 3)

Para el segmento ínfero lateral basal, la variación estuvo dada por subestimaciones de la motilidad; en dos pacientes con motilidad normal por resonancia magnética cardiaca, fue subestimada en el ecocardiograma transtorácico como aquinesia en uno y en otro como hipoquinesia. A excepción del segmento lateral apical, todos los segmentos presentaron solo variaciones asociadas a la subestimación de la motilidad, en promedio alrededor del $13 \%$. Solo el segmento antero lateral basal tuvo una variación del $8 \%$ respecto a la resonancia magnética cardiaca.

\section{Arteria coronaria derecha}

Para el territorio de la arteria coronaria derecha (RCA, su sigla en inglés), el coeficiente kappa global fue bueno (k: $64 \%$ IC. 15), sin embargo, al evaluar los segmentos inferior basal e inferior medio los coeficientes kappa fueron moderados, $45 \%$ y $53 \%$ respectivamente (fig. 4 )

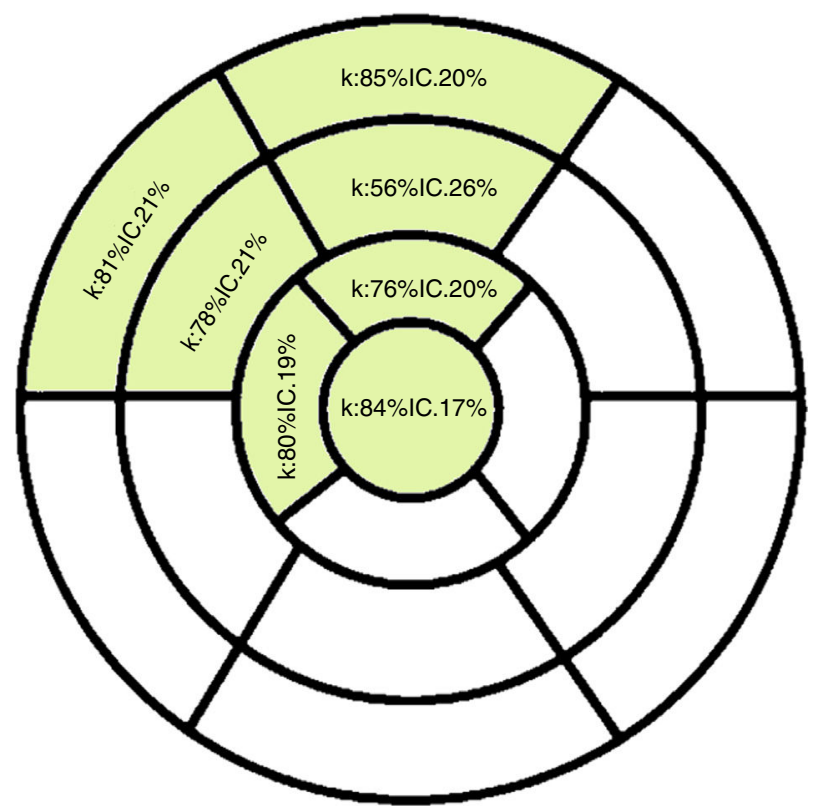

Figura 2 Concordancia de los diagnósticos de motilidad de segmentos irrigados por ADA realizados por ecocardiograma transtorácico y resonancia magnética cardiaca ( $\mathrm{k}$ : valor calculado de kappa; IC: intervalo de confianza para el coeficiente kappa).

Fuente: Datos del estudio.

Los segmentos inferior basal y medio presentaron la mayor variabilidad, lo cual se explica porque en estos la subestimación de la motilidad fue mayor que en todos los segmentos evaluados, $18 \%$ y $16 \%$ respectivamente. Aunque en el segmento inferior apical también hubo subestimación, este no afectó los valores de kappa porque solo se dio en el $2 \%$ de los datos para este segmento.

Para el segmento inferior medio, el $18 \%$ de los pacientes tuvo resonancia magnética cardiaca con motilidad normal, no obstante, por ecocardiograma transtorácico fue clasificado como hipoquinesia o aquinesia, siendo mayor la proporción clasificada para esta última (13\%). El segmento inferior basal presentó el mismo fenómeno de subestimación de la motilidad normal a aquinesia o hipoquinesia.

\section{Global}

En cuanto a la concordancia global, el valor obtenido fue k: $39,45 \%$ IC $6 \%$, valor que se clasifica en aceptable. Esto responde a las variaciones entre los territorios, sin embargo, como valor global y dado que es una técnica de diagnóstico, el valor obtenido no fue ideal (tabla 3 ).

Al revisar detalladamente, se observa que el $62,32 \%$ de los diagnósticos realizados por ecocardiograma 


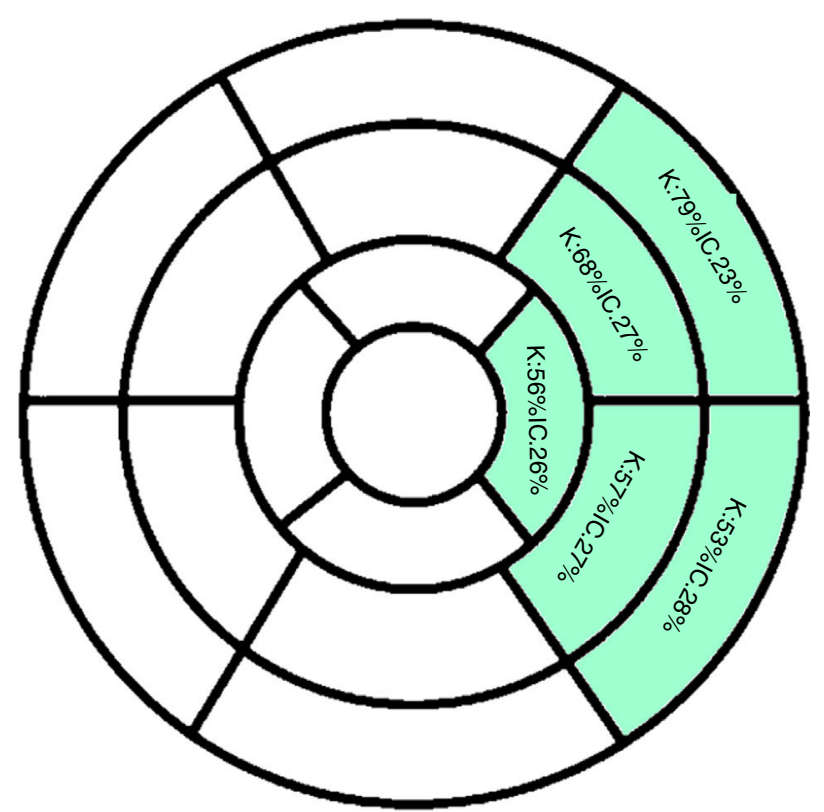

Figura 3 Concordancia de los diagnósticos de motilidad de segmentos irrigados por LCX realizados por ecocardiograma transtorácico y resonancia magnética cardiaca ( $\mathrm{k}$ : valor calculado de kappa; IC: intervalo de confianza para el coeficiente kappa).

Fuente: Datos del estudio.

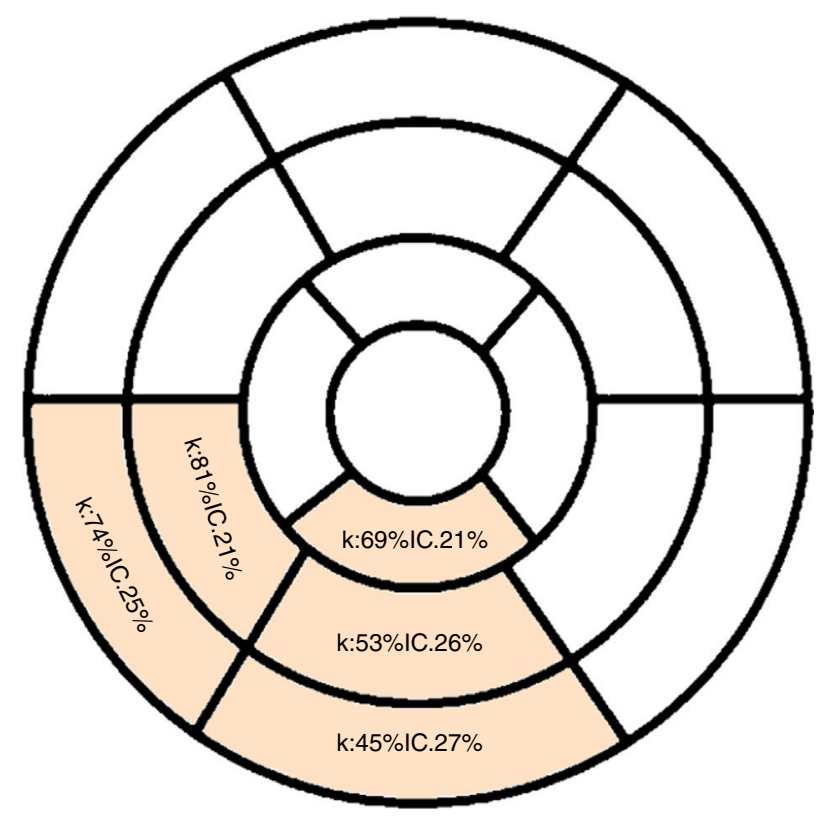

Figura 4 Concordancia de los diagnósticos de motilidad de segmentos irrigados por ACD realizados por ecocardiograma transtorácico y resonancia magnética cardiaca $(\mathrm{k}$ : valor calculado de kappa; IC: intervalo de confianza para el coeficiente kappa)

Fuente: Datos del estudio.

transtorácico coincidieron con la resonancia magnética cardiaca, pese a ello, un $12,25 \%$ fue sobreestimado por el ecocardiograma transtorácico. De otro lado, llama la atención que los valores que fueron valorados por resonancia magnética cardiaca como normales, por ecocardiograma transtorácico fueron aquinéticos.

\section{Discusión}

En este estudio se comparó la motilidad segmentaria entre dos métodos aplicados al mismo paciente en un rango de tiempo en el cual no se esperan cambios en la contractilidad global y segmentaria, teniendo en cuenta la capacidad diagnóstica del ecocardiograma transtorácico respecto a la resonancia magnética cardiaca vs. los resultados de la motilidad segmentaria por territorios anatómicos.

Una de las indicaciones más frecuentes de la ecocardiografía y de la resonancia magnética es la cardiopatía isquémica ${ }^{19}$; además en el infarto se acentúa el compromiso segmentario en los territorios afectados, razón por la cual se eligió esta patología como criterio de selección.

Aunque evalúan parámetros similares, la valoración de volúmenes y masa miocárdica depende específicamente de la técnica de imagen utilizada; es así como existen diferentes valores de normalidad para cada una ${ }^{20}$, sin haber diferencia significativa entre ambas técnicas, especialmente en la medición de la FEVI; si bien para la línea basal el cálculo de la FEVI es menor con el ecocardiograma transtorácico que en la resonancia magnética cardiaca, no se ha encontrado evidencia que uno sea mejor que otro respecto a este parámetro ${ }^{21}$.

El ecocardiograma transtorácico es la técnica más usada en la actualidad para la valoración tanto de la fracción de eyección como de la motilidad segmentaria, la cual acumula varios años de experiencia y cuenta con múltiples trabajos que avalan su uso ${ }^{22}$. En este estudio, al comparar ambos métodos, la cuantificación de la fracción de eyección diferenciada entre género, no evidenció diferencia significa para la FEVI, demostrándose así la utilidad y eficiencia del ecocardiograma transtorácico, cuando de hace de manera correcta.

En la evaluación de la motilidad segmentaria, existe una correlación segmentaria adecuada en los segmentos de la pared septal y en la pared anterolateral, que muestra concordancia según la correlación por coeficiente de kappa.

En la correlación segmento a segmento entre ambas técnicas, aquellos que muestran mayores diferencias y no alcanzan umbrales significativos de correlación se encuentran en la pared inferior en los segmentos mediobasales, en la pared inferolateral en todos sus segmentos y en la pared anterior medial sin extenderse al segmento basal o apical. La correlación entre ambos métodos para la evaluación de la motilidad segmentaria es débil ${ }^{10}$.

En orden, los territorios arteriales que tuvieron menor concordancia corresponden a los territorios supuestos de las arterias coronaria derecha, diagonal y circunfleja, lo cual se explica por la facilidad de obtención de planos de la resonancia magnética cardiaca, mientras que el ecocardiograma transtorácico depende de la pericia del operador, que aun así se puede ver afectado por la calidad de la ventana acústica que se obtiene con el ecógrafo ${ }^{20}$.

Al igual que en el presente estudio, Gadner et al. ${ }^{21}$ encontraron una diferencia en los valores netos de evaluación de FEVI, pero la diferencia entre estos no fue significativa, lo cual da a entender que la utilización del ecocardiograma transtorácico para la obtención de la FEVI, con un correcto entrenamiento del operador, es útil para el diagnóstico y manejo de los pacientes.

La ecocardiografía es el método más usado para la valoración cardiológica en diferentes centros hospitalarios, es económica y versátil ${ }^{19}$, aunque su obtención e 
Tabla 3 Diagnósticos de motilidad de todos los segmentos

\begin{tabular}{|c|c|c|c|c|c|c|}
\hline \multirow[t]{2}{*}{ Diagnósticos de motilidad segmentaria } & & \multicolumn{3}{|c|}{ EcoTT } & \multirow[b]{2}{*}{ Disquinesia } & \multirow[t]{2}{*}{ Total } \\
\hline & & Aquinesia & Hipoquinesia & Normal & & \\
\hline \multirow[t]{4}{*}{ Resonancia magnética cardiaca } & Aquinesia & $10,39 \%$ & $4,81 \%$ & $7,44 \%$ & $0,00 \%$ & $22,64 \%$ \\
\hline & Hipoquinesia & $4,96 \%$ & $14,26 \%$ & $4,81 \%$ & $0,00 \%$ & $24,03 \%$ \\
\hline & Normal & $2,33 \%$ & $12,09 \%$ & $37,67 \%$ & $0,00 \%$ & $52,09 \%$ \\
\hline & Disquinesia & $1,09 \%$ & $0,16 \%$ & $0,00 \%$ & $0,00 \%$ & $1,24 \%$ \\
\hline Total & & $18,76 \%$ & $31,32 \%$ & $49,92 \%$ & $0,00 \%$ & $100,00 \%$ \\
\hline
\end{tabular}

ECO-TT: Ecocardiograma transtorácico; Aquinesia: Falta, pérdida o ausencia de movimiento en el segmento; Hipoquinesia: Disminución del movimiento o de la actividad funcional del segmento; Normal: Movimiento o de la actividad funcional del segmento dentro de los parámetros estandarizados;

Disquinesia: Movimiento anormal debido a una excesiva y/o inapropiada actividad contráctil del segmento.

Fuente: Datos del estudio

interpretación es operador-dependiente y es poco común realizar control de calidad a los estudios adquiridos; adicionalmente, por su versatilidad, tiene inconvenientes en la adquisición de imágenes por factores implícitos del paciente como obesidad, patologías que deforman la caja torácica, estudios realizados en $\mathrm{UCl}$, decúbito supino y ventilación mecánica asociada a la falta del uso de contraste ecocardiográfico en nuestro medio, los cuales generan ventanas acústicas limitadas. Estos factores no se encuentran en la resonancia cardíaca ${ }^{22}$; sin embargo, esta tiene factores propios que impiden su difusión, como la limitada disponibilidad de equipos, la escasa disponibilidad de personal entrenado en obtención e interpretación de imágenes y los costos.

La resonancia magnética cardíaca tiene una resolución espacial mejor que la del ecocardiograma y se sobrepone a las limitaciones técnicas de este último; permite separar mejor los segmentos de las diferentes paredes, observando defectos que fueron reportados como normales por el ecocardiograma ${ }^{22}$. En este estudio no se evaluaron las razones técnicas por las cuales el ecocardiograma no se correlaciona con la resonancia magnética cardíaca en los segmentos previamente mencionados.

Estas diferencias en el análisis de la motilidad segmentaria pueden ser más marcadas en la adquisición de imágenes de estrés, en las que el ecocardiograma solo evalúa la motilidad segmentaria como marcador de isquemia, creando la posibilidad de falsos negativos en los territorios supuestos de las arterias coronaria derecha, circunfleja y diagonales. Esto podría explicarse como una consecuencia de la revascularización, ya que la motilidad segmentaria se comporta con marcador subrogado al daño tisular.

Una recomendación para próximas evaluaciones, es la toma de datos antes de una capacitación a fin de analizar observaciones previas y posteriores a ésta, mediante cotejos.

En conclusión, la valoración de la fracción de eyección por ecocardiograma no difiere de aquella por resonancia magnética cardíaca. En la valoración de la motilidad segmentaria la resonancia magnética cardíaca es superior al ecocardiograma.

\section{Financiación}

Centro de Investigaciones Clínica de la Costa.

\section{Conflicto de intereses}

Ninguno.

\section{Bibliografía}

1. Moreno PR, del Portillo JH. Isquemia miocárdica: conceptos básicos, diagnóstico e implicaciones clínicas Primera parte. Rev Colomb Cardiol. 2016;23:403-9, http://dx.doi.org/ 10.1016/j.rccar.2016.06.009.

2. Moreno PR, del Portillo JH. Isquemia miocárdica: conceptos básicos, diagnóstico e implicaciones clínicas Segunda parte. Rev Colomb Cardiol. 2016;23:500-7, http://dx.doi.org/10. 1016/j.rccar.2016.01.024.

3. Moreno PR, del Portillo JH. Isquemia miocárdica: conceptos básicos, diagnóstico e implicaciones clínicas Tercera parte. Rev Colomb Cardiol. 2017;24:34-9, http://dx.doi.org/10. 1016/j.rccar.2016.02.005.

4. Lang RM, Badano LP, Mor-Avi V, Afilalo J, Armstrong A, Ernande $\mathrm{L}$, et al. Recommendations for cardiac chamber quantification by echocardiography in adults: an update from the American Society of Echocardiography and the European Association of Cardiovascular Imaging. J Am Soc Echocardiogr. 2015;28, http://dx.doi.org/10.1016/j.echo.2014.10.003, 1-39.e14.

5. Cerqueira MD, Weissman NJ, Dilsizian V, Jacobs AK, Kaul S, Laskey WK, et al. Standardized myocardial segmentation and nomenclature for tomographic imaging of the heart. A statement for healthcare professionals from the Cardiac Imaging Committee of the Council on Clinical Cardiology of the American Heart Association. Circulation. 2002;105:539-42.

6. Medrano GA, Aranda A, Meléndez G, de Micheli A. On the actual nomenclature of myocardial infarcts. Arch Cardiol Mex. 2010;80:126-32.

7. Chuang ML, Hibberd MG, Salton CJ, Beaudin RA, Riley MF, Parker RA, et al. Importance of imaging method over imaging modality in noninvasive determination of left ventricular volumes and ejection fraction: assessment by two- and three-dimensional echocardiography and magnetic resonance imaging. J Am Coll Cardiol. 2000;35:477-84.

8. Boyd AC, Schiller NB, Thomas L. Principles of transthoracic echocardiographic evaluation. Nat Rev Cardiol. 2015;12:426-40, http://dx.doi.org/10.1038/nrcardio.2015.57.

9. Lieberman AN, Weiss JL, Jugdutt BI, Becker LC, Bulkley BH, Garrison JG, et al. Two-dimensional echocardiography and infarct size: relationship of regional wall motion and thickening 
to the extent of myocardial infarction in the dog. Circulation. 1981;63:739-46.

10. Nowosielski M, Schocke M, Mayr A, Pedarnig K, Klug G, Köhler $A$, et al. Comparison of wall thickening and ejection fraction by cardiovascular magnetic resonance and echocardiography in acute myocardial infarction. J Cardiovasc Magn Reson. 2009;11:22, http://dx.doi.org/10.1186/1532-429X-11-22.

11. Friedrich MG, Bucciarelli-Ducci C, White JA, Plein S, Moon JC, Almeida AG, et al. Simplifying cardiovascular magnetic resonance pulse sequence terminology. J Cardiovasc Magn Reson. 2014;16:3960, http://dx.doi.org/10.1186/s12968-014-0103-z.

12. Jenkins C, Bricknell K, Hanekom L, Marwick TH. Reproducibility and accuracy of echocardiographic measurements of left ventricular parameters using real-time three-dimensional echocardiography. J Am Coll Cardiol. 2004;44:878-86, http://dx.doi.org/ 10.1016/j.jacc.2004.05.050.

13. O'Gara PT, Kushner FG, Ascheim DD, Casey DE, Chung MK, de Lemos JA, et al. 2013 ACCF/AHA Guideline for the Management of ST-Elevation Myocardial Infarction: A Report of the American College of Cardiology Foundation/American Heart Association Task Force on Practice Guidelines. Circulation. 2013;127(4):e362-425, https://doi.org/ 10.1161/CIR.0b013e318f62742.

14. Ortiz-Pérez JT, Rodríguez J, Meyers SN, Lee DC, Davidson C, Wu E. Correspondence between the 17-segment model and coronary arterial anatomy using contrastenhanced cardiac magnetic resonance imaging. JACC Cardiovasc Imaging. 2008;1:282-93, http://dx.doi.org/ 10.1016/j.jcmg.2008.01.014.

15. Picard MH, Adams D, Bierig SM, Dent JM, Douglas PS, Gillam LD, et al. American Society of Echocardiography Recommendations for Quality Echocardiography Laboratory Operations. J Am Soc Echocardiogr. 2011;24:1-10, http://dx.doi.org/ 10.1016/j.echo.2010.11.006.
16. Chisholm CB, Dodge WR, Balise RR, Williams SR, Gharahbaghian L. Beraud A-S. Focused Cardiac Ultrasound Training: How Much Is Enough? J Emerg Med. 2013;44:818-22, http://dx.doi.org/10.1016/j.jemermed.2012.07.092.

17. Moncho Vasallo J, Nolasco Bonmatí A. Conceptos básicos de estadística descriptiva y probabilidad. En: Vasallo JM, Bonmatí AN, editores. Estadística Aplicada a Las Ciencias de La Salud. Barcelona (Esp):. Elsevier; 2015. p. 1-44, http://dx.doi.org/10.1016/B978-84-9022-446-5.00001-X.

18. McHugh ML. Interrater reliability: the kappa statistic. Biochem Medica. 2012;34:276-82, http://dx.doi.org/10. 11613/BM.2012.031.

19. Díaz Águila HR. Clinical echography: What? who? what for? Medwave. 2016;16, http://dx.doi.org/10.5867/medwave. 2016.08.6547, e6547-e6547.

20. Duncan RF, Dundon BK, Nelson AJ, Pemberton J, Williams $\mathrm{K}$, Worthley MI, et al. A study of the 16-Segment Regional Wall Motion Scoring Index and biplane Simpson's rule for the calculation of left ventricular ejection fraction: a comparison with cardiac magnetic resonance imaging. Echocardiography. 2011;28:597-604, http://dx.doi.org/ 10.1111/j.1540-8175.2011.01394.x.

21. Gardner BI, Bingham SE, Allen MR, Blatter DD, Anderson JL. Cardiac magnetic resonance versus transthoracic echocardiography for the assessment of cardiac volumes and regional function after myocardial infarction: an intrasubject comparison using simultaneous intrasubject recordings. Cardiovasc Ultrasound. 2009;7:38, http://dx.doi.org/10.1186/1476-7120-7-38.

22. Arias T, Chen J, Fayad ZA, Fuster V, Hajjar RJ, Chemaly ER. Comparison of echocardiographic measurements of left ventricular volumes to full volume magnetic resonance imaging in normal and diseased rats. J Am Soc Echocardiogr. 2013;26:910-8, http://dx.doi.org/10.1016/j.echo.2013.04.016. 